Int. J. Dev. Biol. 57: 13-24 (2013)

doi: $10.1387 / \mathrm{ijdb} .120111 \mathrm{ap}$

\title{
In vivo imaging of Drosophila wing heart development during pupal stages
}

\author{
MARKUS TÖGEL ${ }^{\#, 1,2}$, GÜNTHER PASS ${ }^{2}$ and ACHIM PAULULAT*,1 \\ ${ }^{1}$ Department of Biology, University of Osnabrück, Zoology/Developmental Biology, Osnabrück, Germany and \\ ${ }^{2}$ Department of Evolutionary Biology, University of Vienna, Vienna, Austria
}

\begin{abstract}
Wing hearts are small pumping organs that maintain the flow of hemolymph through the wing veins of insects. In Drosophila, these organs consist of parallel oriented muscle cells and a simple epithelium of connective tissue. Both tissues originate from eight embryonic wing heart progenitors (WHPs), which remain dormant until late larval stages. Most of the differentiation and maturation takes place during the pupal stage following head eversion. In this study, we have used the tissue specific expression of Gal4 enhancer lines, in combination with the live cell markers GFP and DsRed to investigate pupal wing heart development in conjunction with the surrounding tissues. We found that WHPs interact with the tracheal system and specific expression domains of the adult epidermis. Additionally, wing heart development occurs simultaneously with the remodeling of the dorso-lateral epidermis into the scutellum and the scutellar arms. Myogenesis in wing hearts comprises known processes such as founder cell specification, but also new features like removal of growing myotubes, and nuclei movement. Wing heart epithelium development is accomplished by the mesenchymal-epithelial transition of WHPs and occurs slightly delayed to muscle development. The epithelium represents a novel mesodermally derived secondary epithelium. Moreover, we have identified a nerve that runs along the epithelium and innervates the wing heart muscle cells.
\end{abstract}

KEYWORDS: adultmyogenesis, nuclearmigration, pannier, engrailed, circulatory organ, mesenchymal-epithelial transition

\section{Introduction}

In the open circulatory system of insects, the dorsal vessel (heart proper) is supported by accessory pulsatile organs that facilitate hemolymph exchange in long and narrow body appendages, e.g., the wings (Pass, 2000, Pass et al., 2006). Although wings consist mostly of dead cuticle, they contain living cells lining their veins and thus require supply with hemolymph. The accessory pulsatile organs that specifically maintain circulation through the narrow wing veins, evolved in the dorsal part of the thorax and are located in a tergal elevation called the scutellum. Comparative investigations revealed that these wing circulatory organs exhibit a great morphological diversity among winged insects (Krenn and Pass, 1995). Drosophila has bilateral wing circulatory organs located at the lateral corners of the scutellum where the scutellar arms, the tubular connections to the posterior wing veins, insert. Since they are functionally independent of the dorsal vessel, they are referred to as wing hearts. Each wing heart consists of about seven parallel muscle cells and an epithelium of connective tissue that separates the muscle cells from the thoracic cavity (Lehmacher et al., 2009). We have previously shown that these organs and both types of tissues originate from a total of eight embryonic progenitors. These wing heart progenitors (WHPs) arise as a subset of the even-skipped positive pericardial cells (EPCs) by down-regulation of tinman expression and represent a new set of adult progenitors. EPCs are counted among the cardiac mesoderm due to their spatial proximity to the heart and tinman expression. However, WHPs share many similarities with adult muscle precursors (AMPs) and the mature wing heart muscles resemble adult somatic muscles. All eight WHPs are interconnected via cellular processes and four

Abbreviations used in this paper: AMP, adult muscle precursor; WHP, wing heart progenitor.

\footnotetext{
*Address correspondence to: Achim Paululat. Department of Biology, University of Osnabrück, Zoology/Developmental Biology, Barbarastraße 11, D-49069 Osnabrück, Germany. Fax: +49.541.969.2587. e-mail: Paululat@Biologie.Uni-Osnabrueck.DE
}

\#Current address: The Weatherall Institute of Molecular Medicine, University of Oxford, John Radcliffe Hospital, Headington, Oxford OX3 9DS, UK

Supplementary Material (6 movies) for this paper is available at: http://dx.doi.org/10.1387/ijdb.120111ap 
of them are associated with the dorsal tracheal branches of the second and third thoracic segment. After their specification in the embryo, WHPs remain dormant until the transition from the second to the third larval instar when proliferation is initiated leading to eight clusters of WHPs. During the prepupal stage, all WHPs are relocated together with the tracheal branches into a single large cluster of cells located in the dorsal midline in the posterior third of the second thoracic segment. With the onset of the pupal stage, following head eversion, the WHPs spread laterally and give rise to the muscle cells and the epithelium (Tögel et al., 2008). This suggests that the differentiation and maturation process takes place after head eversion during the pupal stage.

Herein, we have focused on the developmental stages following the relocation into a single cluster, which comprise the lateral spreading of WHPs, specification of muscle and epithelial cells, and the formation of these tissues. We were especially interested in studying these developmental processes in the context of the surrounding tissues to identify possible interactions. We confirm that WHPs are associated with the tracheal branches until head eversion. They interact with the overlying adult epidermis and reside in specific expression domains. Additionally, wing heart development is coordinated with the remodeling of the tergal epidermis during formation of the scutellum and the scutellar arms. Like in adult somatic myogenesis, wing heart muscle development is preceded by founder cell specification. However, it also shows unique features that include the removal of growing myotubes and extensive nuclei movement during maturation. The wing heart epithelium is formed by mesenchymal-epithelial transition of WHPs and its development is delayed with respect to muscle formation. Tight cell-cell contacts are established after myogenesis is completed. Additionally, we were able to identify a nerve that projects motor neurons towards the wing heart.

\section{Results}

\section{Wing hearts develop in concert with the formation of the scutellum}

At about 3.5 h APF, eversion of the thoracic imaginal discs takes place and the adult epidermal cells subsequently spread from the discs to replace the larval epidermis. At about 6 h APF, the newly formed mesothoracic epidermis has fused in the dorsal midline and by 10-11 h APF also with the head and the larval epidermis of the abdomen. In contrast, the adult epidermis originating from the prothoracic and metathoracic discs has not spread to the dorsal midline yet. Thus, at this developmental stage, a temporary discontinuity in segment order exists (Fristrom and Fristrom, 1983; Usui and Simpson, 2000). The relocation of the WHPs into one large cluster coincides with the process of imaginal disc evagination. To investigate pupal wing heart development in relation to the epidermis, we used the ubiquitously expressed act5C-Gal4 line to drive UAS-eGFP in combination with handC-DsRed which marks all WHPs. See Materials and Methods section and Fig. 1 for details of the experimental setup. Shortly after the transition from prepupa to pupa (about $12 \mathrm{hAPF}$ ), the dorsal epidermis runs almost straight between thorax and abdomen without a visible separation of the thoracic and abdominal segments (Fig. 2A). Laterally, a constriction between thorax and abdomen is already visible. At this stage, the WHPs are still located in one single large cluster in the dorsal midline. During further development, the epidermis undergoes
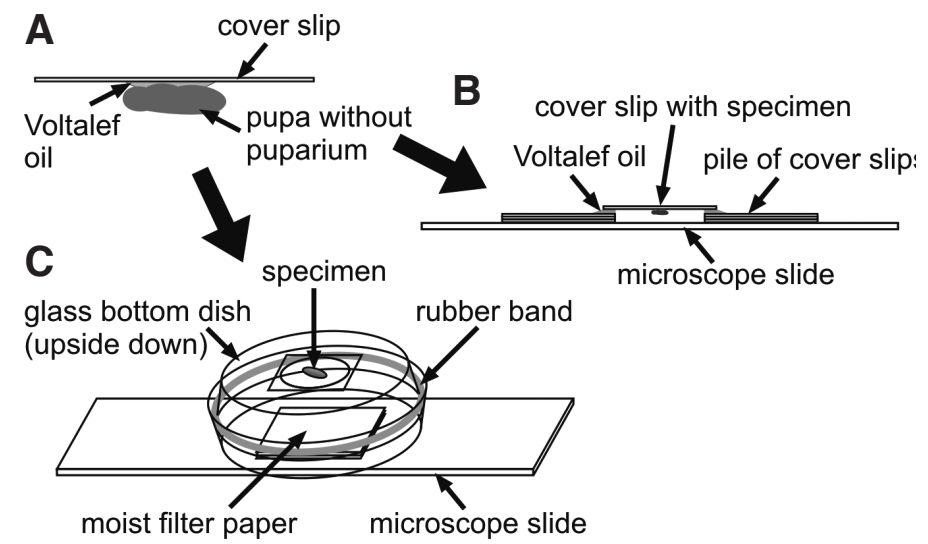

Fig. 1. Preparation of pupae for live cell imaging. (A) To enable the use of oil immersion objectives, pupae without puparium were attached dorsal side up to a cover slip with a small drop of Voltalef oil. This keeps the specimen very close to the cover slip and reduces refraction. (B) For single confocal stacks, the cover slip with the specimen was then mounted using Voltalef oil on top of two piles of cover slips glued to a microscope slide. (C) For time lapse movies, pupae were attached from the inside to the cover slip of a glass bottom dish glued to a microscope slide bottom side up. To prevent dehydration, moist filter paper was put inside the dish and the top and the bottom was sealed by a rubber band.

shape changes that result in the three dimensional structures of the scutellum and its bilateral connections to the posterior wing veins, the scutellar arms. In concert with the reshaping of the epidermis, the WHPs spread laterally and form the wing hearts in the developing lateral corners of the scutellum. At about $20 \mathrm{~h} \mathrm{APF}, \mathrm{a}$ constriction between thorax and abdomen occurs that demarcates the posterior edge of the scutellum. Simultaneously, the outline of the scutellar arms becomes visible on either side of the body (Fig. $2 A)$. During the subsequent development, the constriction gradually extends ventrally and becomes subdivided into compartments. At about 30-40 h APF, the postnotum, the tergal plate posterior to the scutellum, is discernible (Fig. 2A). Between 30-50 h APF, bristles are formed by the outgrowth of the trichogen cells, which gradually elongate to form the shaft, and the tormogen cells, which build the sockets (Fig. 2B). From about 40-50 h APF on, the folding of the dorsal surface of the thorax increases, especially the scutellum exhibits two longitudinal folds and one transverse fold. Since the scutellum extends from the notum at an oblique angle after eclosion and has a greater volume than during pupal stages, it is feasible that the folding occurs due to limited space for the large surface. See also supplemental movie M01 that illustrates the formation of the scutellum and wing heart development.

\section{The scutellar arms are formed by a folding of the lateral tergal epidermis}

The tubular connection between scutellum and posterior wing vein is formed by the lateral epidermis. At about $15 \mathrm{~h}$ APF, the adult epidermis shows uniform thickness in cross sections dorsal to the wing (Fig. 2C). Tube formation starts at about $20 \mathrm{~h} \mathrm{APF}$ with a local thickening in the area of the future scutellar arm just below the lateral corner of the scutellum. Horizontal sections through the thickening show that it represents an outward folding of the epidermis. During the subsequent development, the folding elongates ventrally and a thin lumen becomes visible as dark 
line between the epidermal cells (Fig. 2C). Between 35 and $40 \mathrm{~h}$ APF, brightly fluorescent cells become visible in the middle of the folding extending from the wing to the wing hearts. Based on the used act5C-Gal4 driver line, these cells express high levels of actin suggesting cell shape changes or motility. Time lapse movies show that the brightly fluorescent cells are not static (see supplemental movie M02). They alternate their shape between elongated and round and move within the tube. At about 45-50 h APF, shortly before the wing hearts are mature, the cells become fewer leaving behind a lumen (Fig. 2C). At least the dorsal cells migrate towards the wing heart and then into the body cavity.

WHPs are associated with the pnr-Gal4 expression domain and relocate towards the en-Gal4 expression domain

After the relocation into a single cluster, the WHPs become gradually free from the trachea and attach to the overlying epidermis.
According to Gal4 expression, the epidermis can be subdivided into domains. pannier-Gal4 (pnr-Gal4) driven UAS-eGFP is expressed in the dorsal epidermis with lateral extensions towards the abdomen (Calleja et al., 2000). These lateral patches partly represent the dorsal part of the third thoracic (T3) segment (Fig. 3A). With the appearance of the dorsal constriction between thorax and abdomen, the start of the reshaping of the epidermis, the lateral extensions become shorter. This is due to the dorsal expansion of T3 but also due to the formation of the scutellum. This suggests that these lateral areas of the epidermis constitute the lateral corners of the scutellum where the wing hearts will form. During their entire pupal development, the WHPs are located beneath the pnr-Gal4 expression domain. To assess whether WHPs actively migrate to the lateral corners of the scutellum or are passively transported by the reshaping of the epidermis, we analyzed their movement in relation to the epidermis. As landmarks in the epidermis we used eight of the
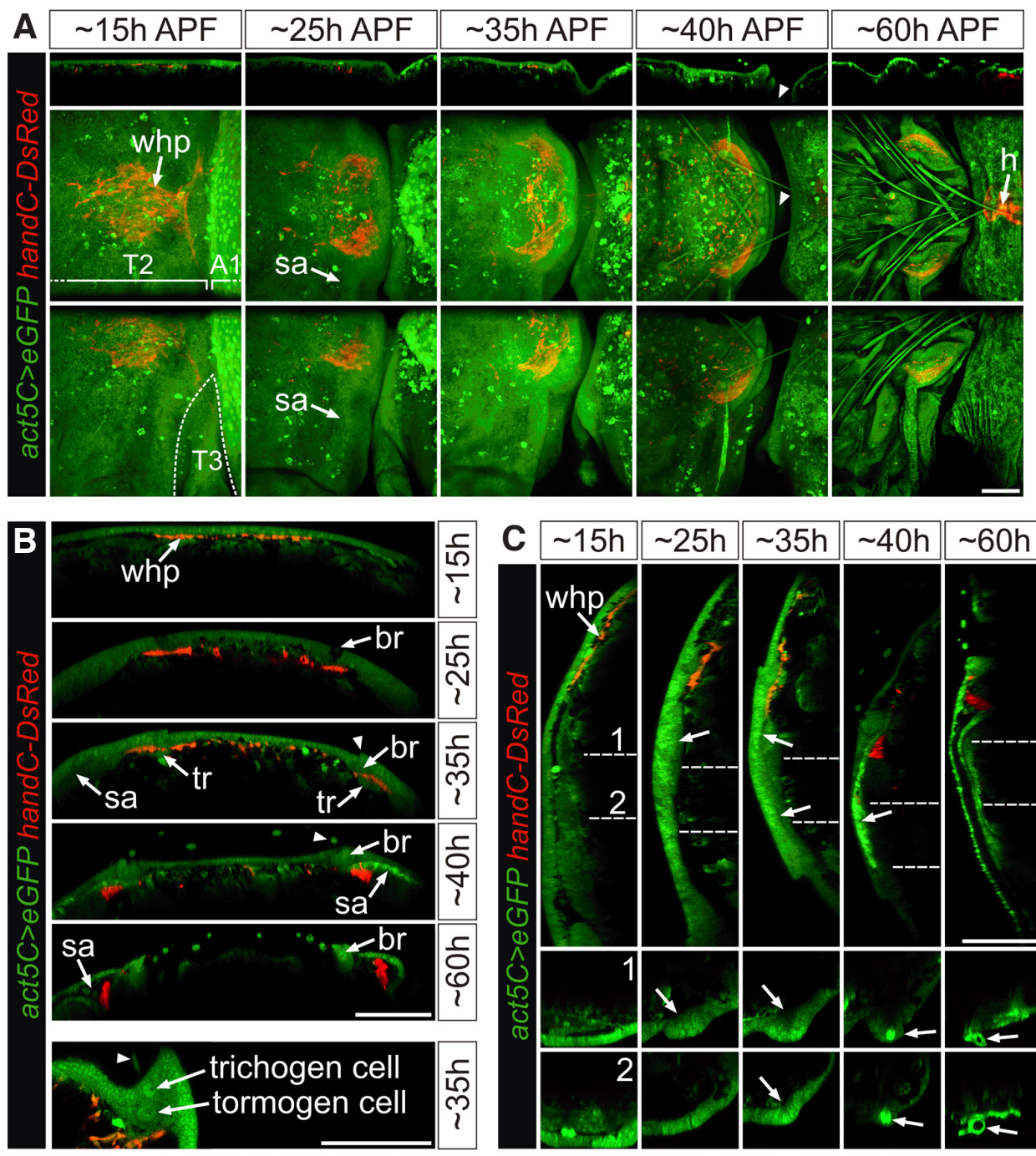

Fig. 2. Wing heart development occurs in concert with scutellum and scutellar arm formation. (A) At each time point a single individual is depicted in dorsal view (middle panel) and dorso-lateral view (lower panel). Additionally a sagittal section through the dorsal view is provided (upper panel). At 15 $h A P F$, wing heart progenitors (whp) form a single large cluster in the dorsal midline of the second thoracic segment (T2). The dorsal epidermis runs straight between thorax and abdomen and the third thoracic segment (T3, outlined by a dashed line) and has not reached the dorsal midline yet leading to a temporal discontinuity in segment order. From about $20 \mathrm{~h}$ APF on, the wing heart progenitors spread laterally, while the adult epidermis is reshaped to form the separation between thorax and abdomen, the scutellum, and the scutellar arm (sa). At later stages, the anterior region of the heart (h) becomes visible below the epidermis. For further illustration see supplementarymovie M01. (B) Cross sections through the dorsal area of the same individuals depicted in $A$. The wing heart progenitors (whp) are tightly attached to the overlying epidermis during their lateral movement towards the forming scutellum. Large bristle cells (br) give rise to bristles (arrowheads) and may be used as landmarks to assess the movement of the wing heart progenitors. An enlargement of this area, shown at the bottom of this panel, illustrates the location of trichogen and tormogen bristle cells. The growing bristle is marked by an arrowhead. The newly forming adult trachea (tr) are tightly attached to the epidermis and reside below the wing heart progenitors at about $35 \mathrm{~h} \mathrm{APF}$. During scutellar arm (sa) formation, brightly fluorescent cells are visible at its center at about $40 \mathrm{~h}$ APF (see also A). (C) Cross sections (upper panel) and horizontal sections (two lower panels) through the dorso-lateral area of the same individuals depicted in A in the region of scutellar arm formation. Dashed lines in the cross sections marked with number 1 or 2 indicate the levels of the horizontal sections. Between 15 and 25 h APF, a local thickening of the epidermis arises (arrow) just below the area where the lateral margins of the scutellum will form. This thickening elongates in ventral direction during the following stages. At about $35 \mathrm{~h}$ APF, a thin lumen is visible (arrows) in which brightly fluorescent cells are present at about $40 \mathrm{~h}$ APF. At about $60 \mathrm{~h} \mathrm{APF,} \mathrm{scutellar} \mathrm{arm} \mathrm{formation} \mathrm{is} \mathrm{completed} \mathrm{resulting} \mathrm{in} \mathrm{a} \mathrm{tubular} \mathrm{connection}$ between wing and scutellum. For further illustration see also supplemental movie M02. Scale bars: $100 \mu \mathrm{m}$. 
bristle forming trichogen cells that are about 10 times larger than the surrounding epidermal cells (Fig. 2B and 3A). At about 15-20 $\mathrm{h}$ APF, most of the WHPs reside inside the area delimited by the trichogen cells. By the end of wing heart development almost all cells have passed beneath the trichogen cells and now lie outside this area. This further supports the idea that the lateral spreading is due to active migration along the epidermis. However, also the epidermis undergoes lateral spreading or stretching as indicated by the increase in distance between the two posterior most bristle cells on either side. Although different animals were analyzed at the respective developmental stages, this change in pattern cannot be accounted for by mere size differences of the pupae.

Another domain is marked by engrailed-Gal4 (en-Gal4) driven

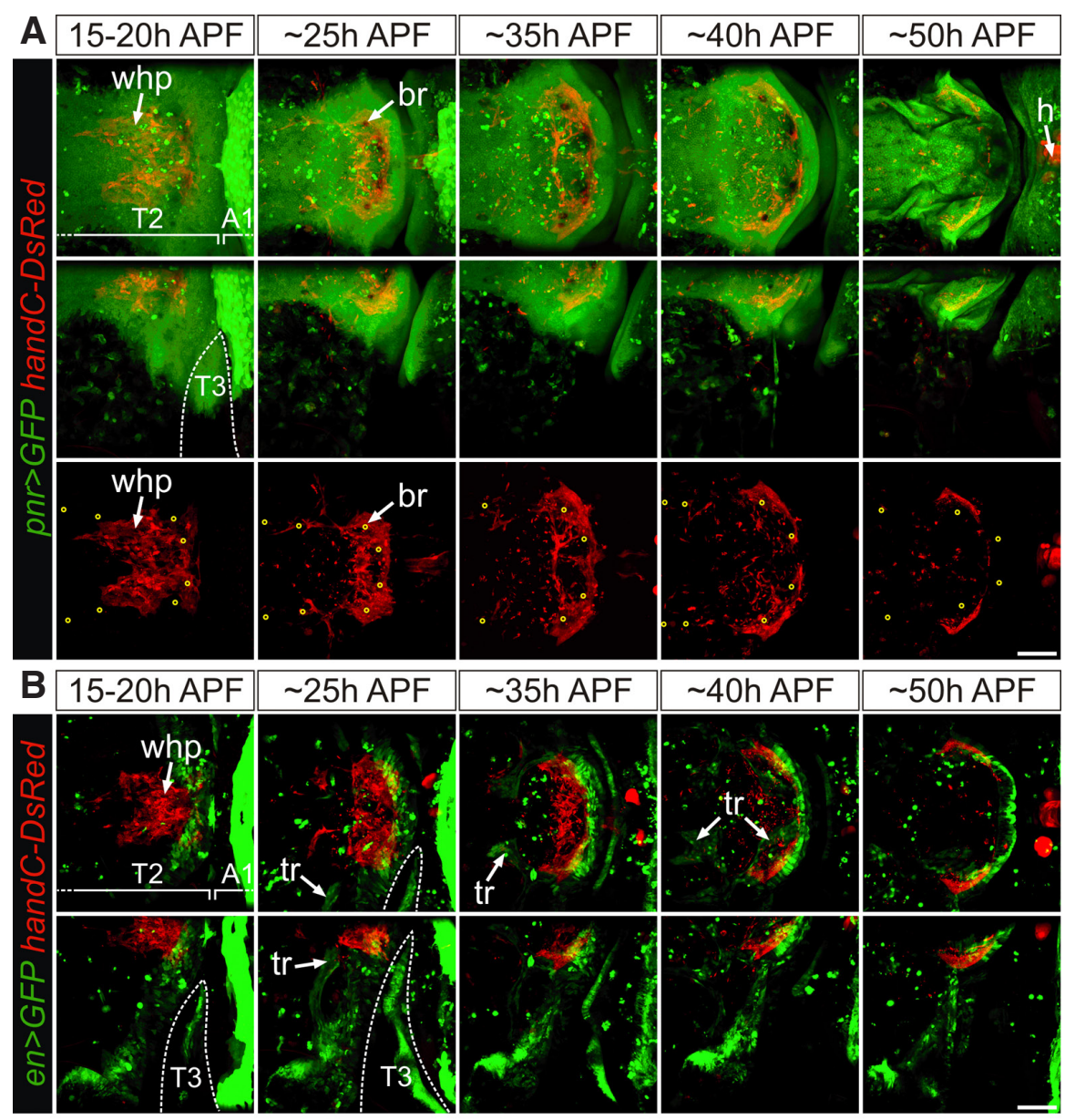

C

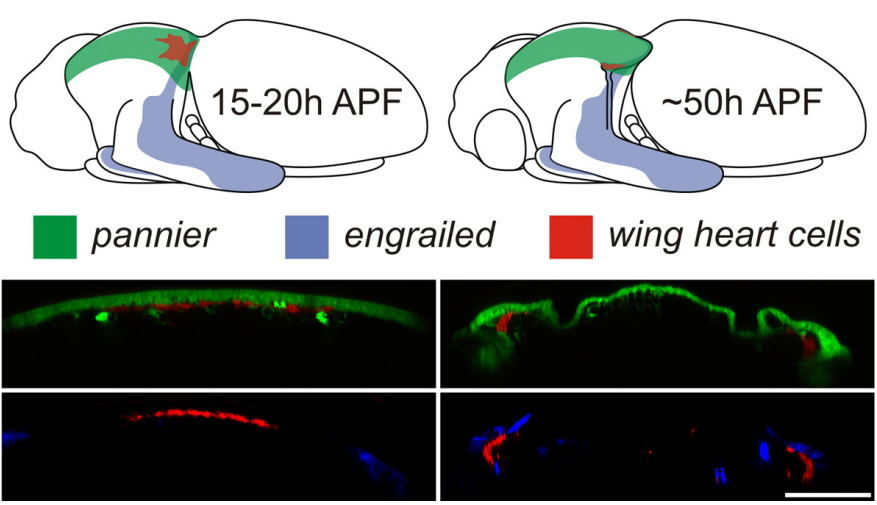

UAS-eGFP, which forms stripes in the posterior region of each segment. At about $15 \mathrm{~h}$ APF, the en-Gal4 expressing domain runs along the posterior margin of the second thoracic segment from the dorsal midline towards the wing (Fig. 3B). Additionally, a weak en-Gal4 expression is detected in the tracheal sacs between 20 and $50 \mathrm{~h}$ APF (Fig. 3B and supplemental movie M03). Initially, the wing heart progenitors lie close but outside the en-Gal4 expressing domain. During scutellum development, the stripe-like appearance of the en-Gal4 expressing domain changes. Like the pnr-Gal4 expressing domain, the domain shifts slightly dorsally and stretches anteriorly. Simultaneously, the WHPs spread laterally. At about 50 $\mathrm{h}$ APF, the entire lateral margin of the scutellum is en-Gal4 positive and the mature wing hearts are completely surrounded by en-Gal4 positive epidermal cells (Fig. 3B and C).

\section{WHPs interact with the tracheal system dur- ing their development}

To specifically visualize the tracheal system, we used breathless-Gal4 (btl-Gal4) to drive UAS-eGFP(see also supplemental movie M04). Till the end of prepupal development, four of the eight clusters of WHPs are closely associated with the interconnection of the dorsal tracheal branches of the second and third thoracic segment. At this stage, most of the WHPs are tightly attached to one another and do not show

Fig. 3. Wing heart development and epidermal domains. (A) The wing heart progenitors (whp) reside within the pnr-Gal4 expression domain during their entire pupal development. Towards the abdomen, the pnr-Gal4 domain exhibits lateral extensions which mark the lateral margin of the future scutellum and the dorsal part of the third thoracic segment (T3). During reshaping of the epidermis and dorsal expansion ofT3, these extensions shorten. Using the position of the large bristle cells (br, yellow circles) as landmarks, the movement of the wing heart progenitors in relation to the epidermis was analyzed. At 15-20 h APF most of the cells are localized within the area demarcated by the bristle cells. In contrast, at about 50 h APF most of the cells are found outside this area. (B) At 15-20 $h A P F$, only the posterior most wing heart progenitors lie within the en-Gal4 expressing domain. Like the pnr-Gal4 domain, the en-Gal4 domain shifts slightly dorsally and stretches anteriorly in the region of the forming lateral margin of the scutellum. At about 50 $h A P F$, the entire wing heart lies beneath en-Gal4 positive epidermal cells. The forming adult trachea (tr) are likewise en-Gal4 positive. Note that the dorsal expansion of the third thoracic segment (T3) is visible by the en-Gal4 stripes that meet in the dorsal midline at about 35 h APF (C) Schematic representations of the pnr and en domains in pupae at about 15-20 h and about $50 \mathrm{~h}$ APF illustrating the relocation of the wing heart progenitors to the lateral overlap of the pnr and en domain during wing heart development. The bottom panel shows the relocation in cross sections at 15-20 h APF (left side) and $50 \mathrm{~h} \mathrm{APF} \mathrm{(right} \mathrm{side).}$ pannier (pnr-Gal4) green channel, engrailed (en-Gal4) blue channel), and wing heart cells (handC-DsRed) red channel. $h$ - heart. Scale bars: $100 \mu \mathrm{m}$. 
A

Fig. 4. Wing heart development and trachea. (A) Anterior region of a third instar larva (L3) illustrating the location of the wing heart progenitors (arrows) in the second (T2) and third (T3) thoracic segments. The posterior wing heartprogenitors (whp) in each segment are associated with the interconnection of the dorsal tracheal branches (tr). The area outlined by the dashed line is enlarged to the right (upper picture shows both channels merged, the picture at the bottom the red channel only). At this stage of development, wing heart progenitors are tightly attached to one another and display a smooth surface. (B) After the wing heart progenitors have become free from the trachea, they lose their tight contact and interconnect via cellular protrusions. At about $20 \mathrm{~h} \mathrm{APF}$, the adult dorsal tracheal supply grows out and branches at about 25 hAPF. One branch forms a tracheal sac inside the scutellum while the other branch forms a tracheal sac for the supply of the indirect flight muscle. At about $40 \mathrm{~h} \mathrm{APF}$, the tracheal sac arches away from the wing heart and only
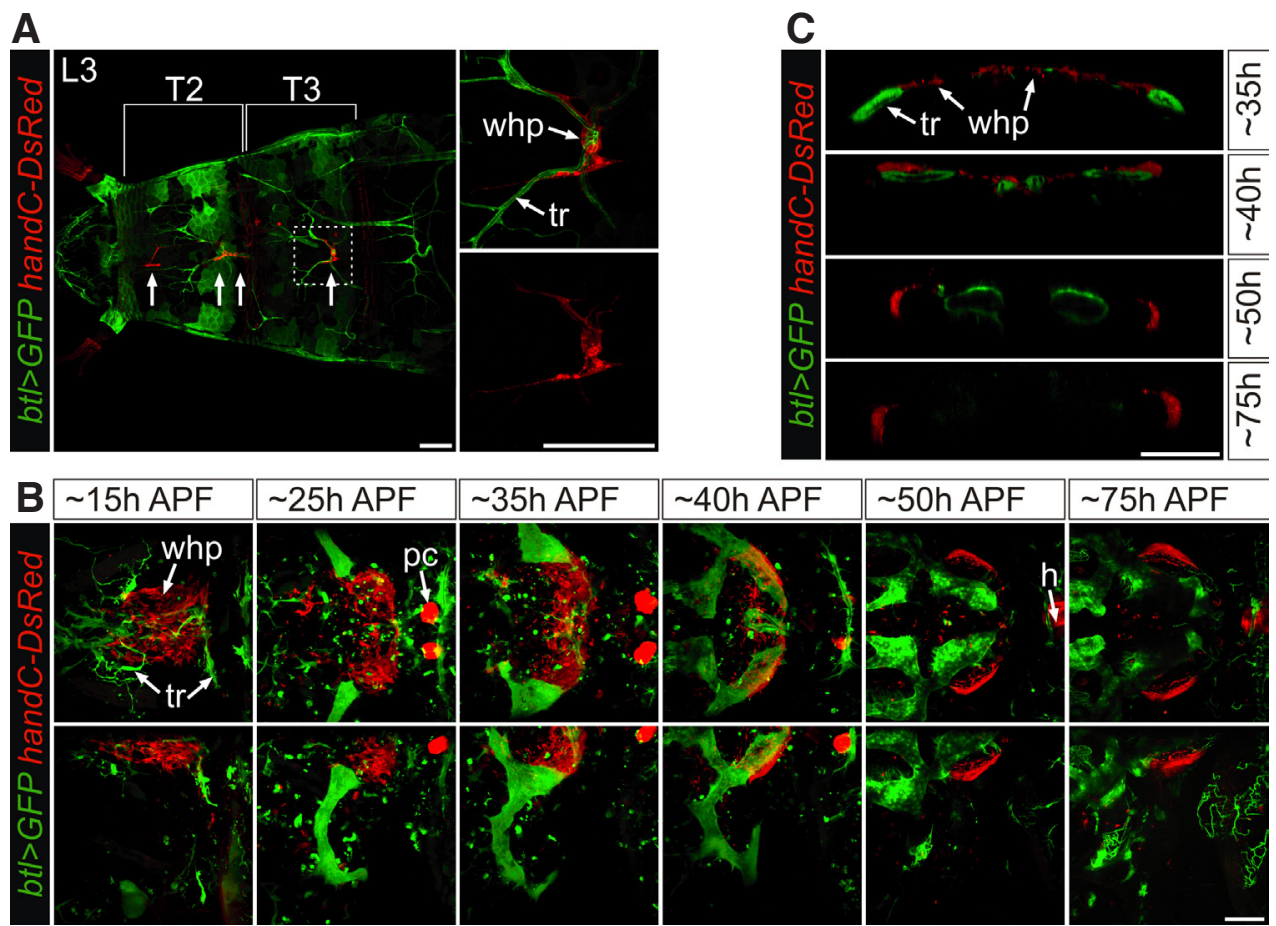

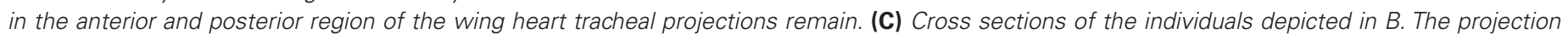

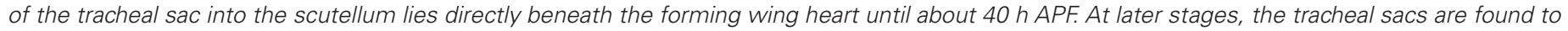

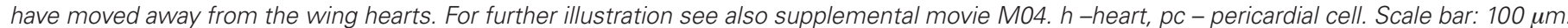

extensive protrusions (Fig. 4A). After their relocation into a single cluster, the WHPs become free from the trachea. At the same time, they lose their tight contact and attach to one another and to the newly formed overlying adult epidermis via multiple cellular protrusions (Figs. 4B, 6E). At about $20 \mathrm{~h} \mathrm{APF}$, the adult tracheal supply of the dorsal mesothorax starts to grow out on either side of the pupa. During their entire development, all new tracheal cells exhibit long cellular protrusions. In contrast to the larval tracheal system, the adult tracheal cells do not form simple tubes in the dorsal mesothorax but large tracheal sacs. The dorsally growing tracheal sacs start to branch at about $25 \mathrm{~h}$ APF as soon as their leading tips reach the anterior edge of the scutellum (Fig. 4B and C). One branch projects into the scutellum and lies immediately beneath the forming wing heart (Fig. 4C) while the other branch extends anteriorly and forms a dorsal tracheal sac supplying the indirect flight muscles (IFM). The branch beneath the wing heart extends fine filopodia towards the wing heart and the epidermis. From about $40 \mathrm{~h}$ APF on, the tracheal sac arches away from the wing hearts and only at the anterior and posterior end of the wing heart projections from the tracheal sac remain. The tracheal supply of the wing heart is probably established by these projections (Fig. 4B). After eclosion of the adult fly the tracheal sacs inside the scutellum inflate and take up the space except for a thin layer of fat body below the epidermis.

Wing heart muscle formation comprises duf-Gal4 expressing starting points, removal of muscle cells, and nuclear movement during maturation

During pupal development, the WHPs become free from the dorsal tracheal branches and establish close contact with the epidermis. By continuous cell divisions they form a pool of similar mesenchymal cells. Cell divisions can be observed almost until the end of wing heart maturation (see supplemental movie M05). It has previously been shown that $d u f-l a c Z$ is expressed in founder cells of adult somatic muscles (Kozopas and Nusse, 2002; Dutta et al., 2004; Fernandes and Keshishian, 2005). Since we wanted to image muscle formation in living animals, we used duf-Gal4, a site specific P-element exchange of the lacZ enhancer trap with a Gal4 P-element, to drive UAS-nDsRed (nuclear localization) as a marker for adult muscle founder cells in combination with handCGFP (nuclear localization) as a marker for WHPs. To identify starting points of muscle formation within the pool of WHPs, we investigated early stages of wing heart development. At 15-18 h APF, a few WHPs were found to express duf-Gal4 but at a very low level (data not shown). However, these cells were not located in the lateral regions where the muscles will form. At about 20-25 h APF, single nuclei expressing duf-Gal4 at moderate levels were found in the lateral regions. In the same animal, we also found one or two duf>DsRed negative nuclei in close contact with one duf $>n D s R e d$ positive nucleus (Fig. 5A). Live imaging confirmed that these small clusters of nuclei represent the starting points of muscle formation. Additional nuclei join these clusters, which then elongate into horizontal rows. In mature organs, up to 17 nuclei are present in a single wing heart muscle cell. During subsequent development, many nuclei of a single cluster (and later row) become $d u f>n D s R e d$ positive. However, the amount of DsRed found in individual nuclei differs considerably (Fig. 5B). Similarly, when using handC-Gal4 driven UAS-mCD8::GFP (membrane GFP) cells are observed that establish close contact and form clusters that elongate into rows (Fig. 6A). Muscle starting points were also detected in the dorsal area where no muscle cells are present in mature organs. In vivo time lapse imaging revealed that these 
developing muscle cells are removed later during development. Cells selected for removal are recognizable by their cell shape change. They round up and the GFP signal becomes fragmented into small bright spots. Shortly thereafter, these spots are removed from the site of wing heart formation (Figs. 5B and 6C).

We observed that the nuclei of the forming myotube are in constant motion. In the initial small clusters, nuclei move around each other, while later in the elongated myotubes, nuclei move along the main axis from anterior to posterior and vice versa in a more coordinated fashion. Especially when reaching the ends of the myotube, nuclei are often compacted into vertical stacks. In contrast, during the movement some nuclei may be elongated or even separated from the remaining nuclei (Fig. 7, and supplementary movie M06). In the mature organ, however, nuclei are more

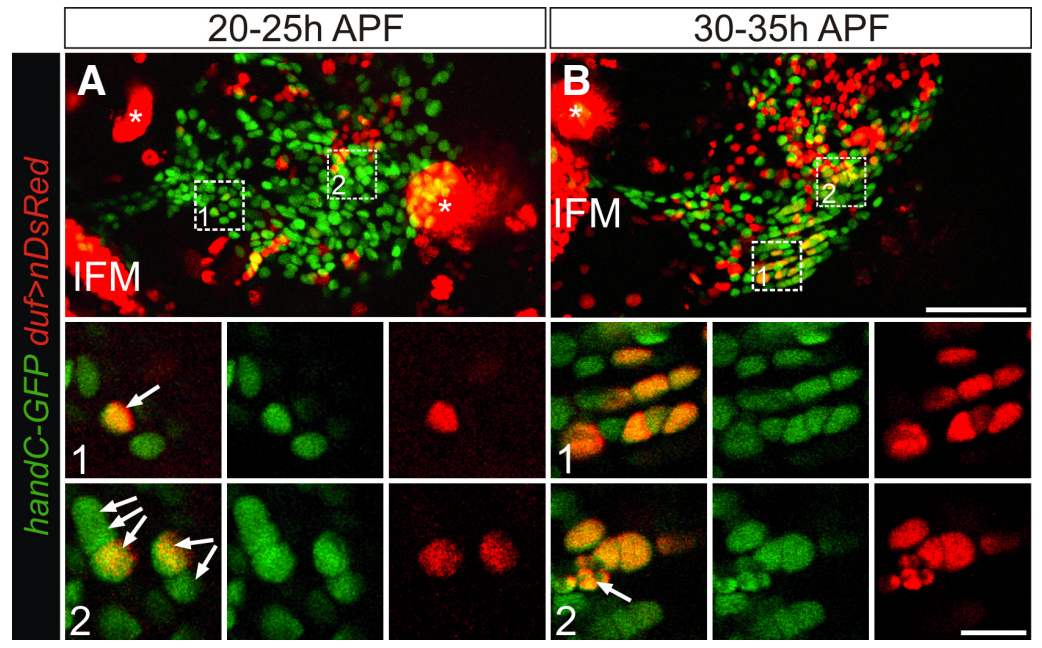

Fig. 5. duf-Gal4 expression in wing heart muscle development. (A) At about 20-25 h APF, single handC-GFP positive nuclei expressing duf-Gal4 at moderate levels are visible in the lateral regions where muscle cells will form. Areas outlined by dashed lines are depicted below at higher magnification. Enlargement 1 shows three separate handC-GFP positive nuclei, of which only one expresses duf-Gal4 (arrow) suggesting that wing heart muscles are seeded by founder cells. Enlargement 2 shows starting points of muscle formation. One or two handC-GFP positive nuclei are tightly attached to each duf-Gal4 positive nucleus (arrows). (B) At later stages, nuclei of muscle cells are arranged in parallel rows and many of them are DsRed positive. Areas outlined by dashed lines are depicted below at higher magnification. Enlargement 1 shows that nuclei within a single row exhibit different amounts of DsRed. Enlargement 2 visualizes the fragmentation of a nucleus (arrow) in a dorsal muscle cells. Asterisks indicate macrophages, IFM - indirect flight muscle. Scale bar: $50 \mu \mathrm{m}$, scale bar in enlargements: $10 \mu \mathrm{m}$.
A

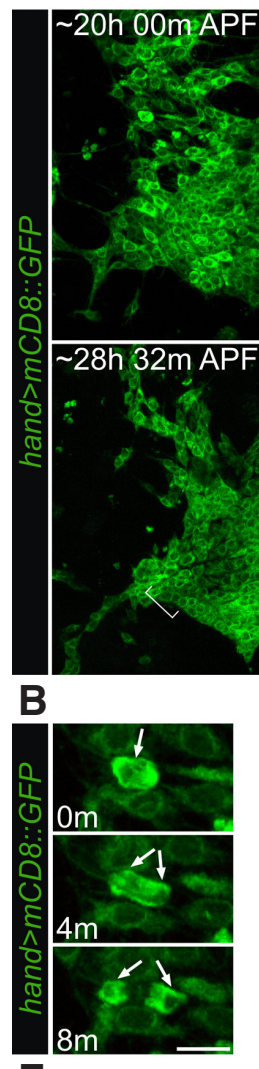

E
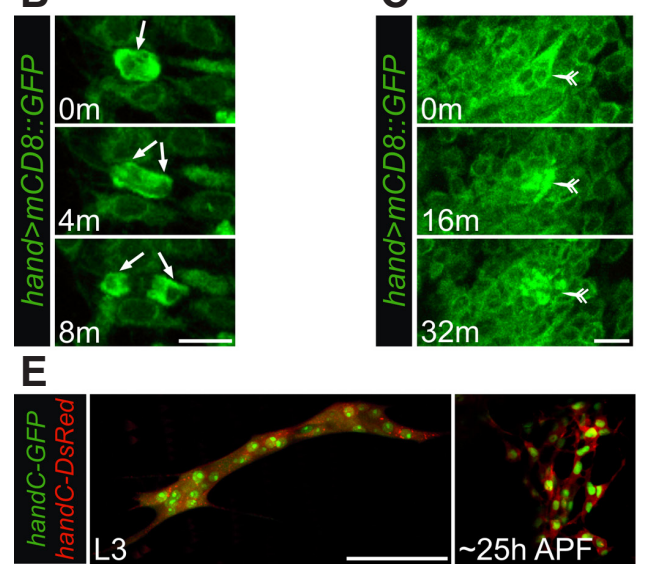

$32 m$
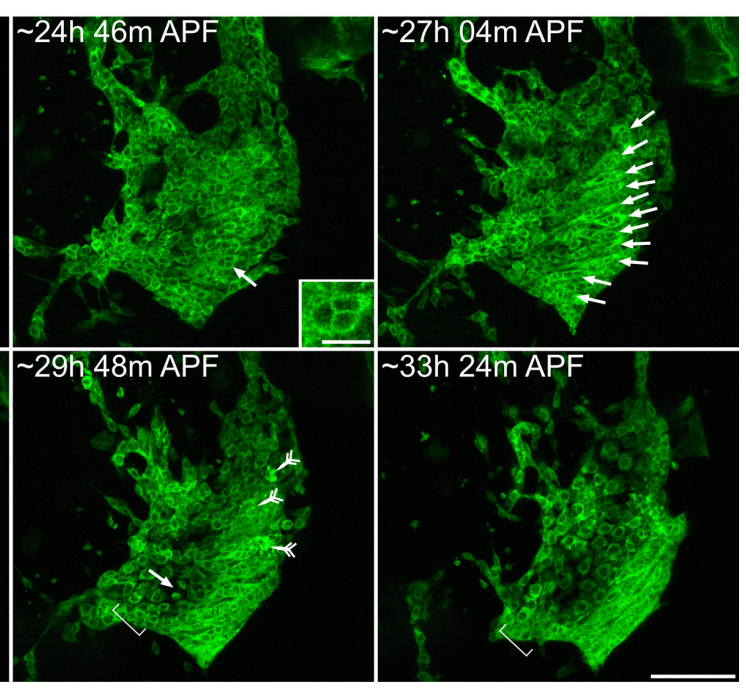

.
Fig. 6.Wing heart muscle and epithelium development. (A) Left wing heart in dorso-lateral view. Depicted are time points taken from a time lapse movie to illustrate the main steps of wing heart muscle and epithelium development (supplementary movie M05). Until about 20 h APF, wing heart progenitors are individual cells which are interconnected by cellular protrusions. Between 20-25 h APF, clusters of two to three cells become visible in the lateral region of the wing heart progenitors (arrow, inset is an enlargement of the cluster), which represent the starting points of muscle formation. Subsequently, the number of starting points increases (arrows in time point 27 h APF) and the clusters contain more nuclei. Muscle formation is also initiated in a dorsal area where no muscle cells are present in the mature organ (compare time points $27 \mathrm{~h}$ and $33 \mathrm{~h}$ APF). These dorsal clusters are removed later during development (flagged arrows in time points 28 $h$ and $29 \mathrm{~h}$ APF). The cells of the wing heart epithelium are recognizable by their larger nuclei, especially in the region that dorsally extends the muscle cells (bracket). As long as the cells have not established a tight contact, cell divisions may occur. At time point $29 \mathrm{~h} \mathrm{APF}$, two small daughter cells are visible in the forming epithelium (arrow). (B-D) Details taken from the time lapse study in A. (B) A dividing wing heart progenitor gives rise to two daughter cells at about $20 \mathrm{~h}$ APF (C) Cluster removal between 28 $h$ and $29 \mathrm{~h}$ APF. The cluster rounds up and the GFP signal becomes fragmented (flagged arrow). (D) The epithelial cells have not established a tight contact until 33 h APF and some cells are removed (arrow). The dashed line indicates the dorsal edge of the wing heart muscles. (E) Wing heart progenitors (visualized by cytoplasmic handC-DsRed and nuclear handC-GFP) display a smooth surface in the cluster at $L 3$ and cellular protrusions after they have become free from the trachea. Scale bar in A: $50 \mu \mathrm{m}$, inset: $10 \mu \mathrm{m}$; in B-D: $10 \mu \mathrm{m}$; in E: $50 \mu \mathrm{m}$. For further illustration see also supplemental movie M05. 
Fig. 7. Nuclear movement during late stages of wing heart muscle development (at about 40 h APF). Depicted are single time points taken from a time lapse movie to itlustrate the intracellularmovement of densely packed nuclei along the main axis of maturating muscle cells (supplementary movie M06). The direction of nuclear movement is indicated by arrows. The wing heart shown consists of six muscle cells (best visible in time point 45 min). Scale bar: $50 \mu \mathrm{m}$.
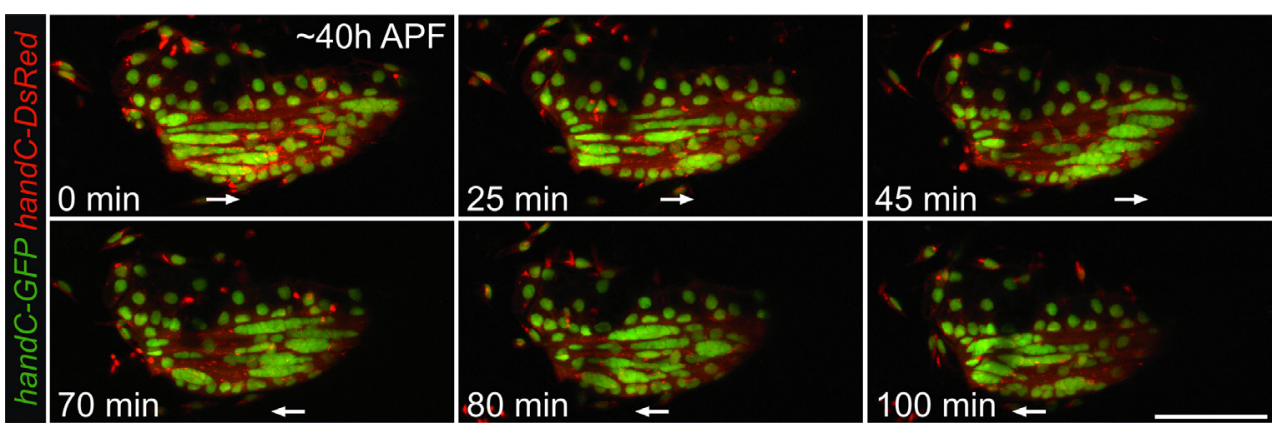

or less equally distributed within the myotube.

Furthermore, we were interested in whether commonly used drivers for targeted gene expression in adult somatic muscles are also active in wing heart progenitors. We tested the expression of Mef2-Gal4 and 24B/how-Gal4 with UAS-eGFP and found that both are expressed during pupal stages (Fig. 8). Remarkably, GFP driven by these lines is not only detected in wing heart progenitors that will later form muscle cells, but also in those that give rise to the non-muscular epithelium. Expression persists in both tissues until the organs are mature (Fig. 8).

To investigate the interaction between epidermal tendon cells and muscle cells during wing heart development we used the stripe-Gal4 line to drive UAS-eGFP. GFP expression becomes first visible in epidermal cells that constitute the anterior and posterior attach-

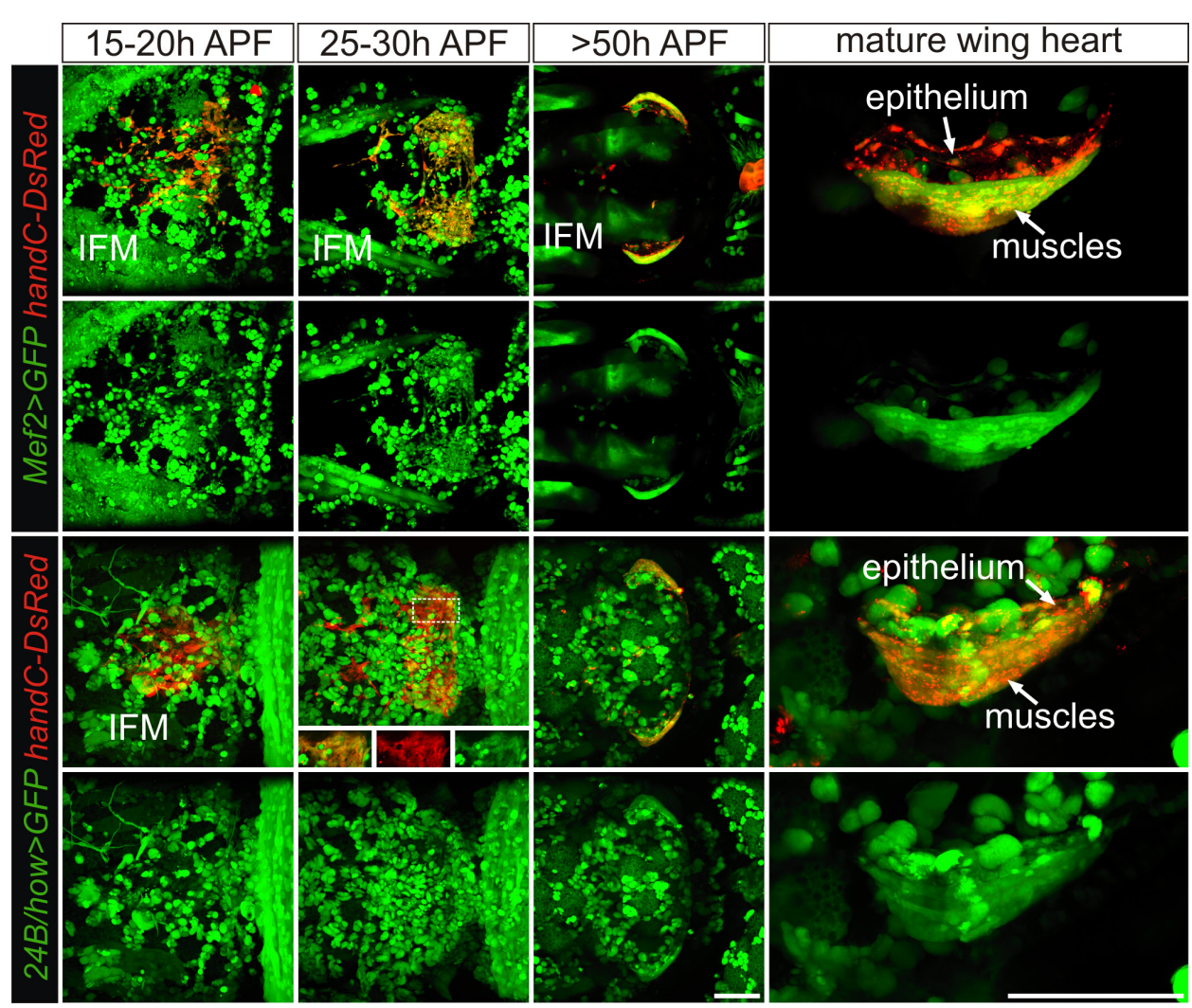

Fig. 8. Expression of muscle specific reporter lines in wing hearts. Depicted are dorsal views of different stages of wing heart development and mature wing hearts in lateral view. Both Mef2-Gal4 and 24B/how-Gal4 are expressed in all wing heart progenitors during the entire pupal development until the organs are mature. The area outlined by dashed lines is depicted as inset to illustrate the overlap in expression in more detail. That the expression is not restricted to the muscles is best seen in mature organs where the epithelium is clearly marked. IFM - indirect flight muscles. Scale bars: $100 \mu \mathrm{m}$. ment sites when muscle cell formation is already completed (Fig. 9). In contrast, the tendon cells of the indirect flight muscles (IFM) are visible throughout the entire IFM development. Furthermore, expression levels differ between the epidermal wing heart tendon cells and not all cells are marked by the reporter. For instance, the dorsal most two muscle cells attach at GFP negative epidermal cells at their posterior end (Fig. 11B).

\section{Wing heart epithelium formation occurs slightly delayed to muscle formation}

A subset of WHPs undergoes mesenchymal-epithelial transition to form the wing heart epithelium. Before about 20-25 h APF, these cells are morphologically indistinguishable from those that will give rise to the wing heart muscle cells and may not even be specified yet. However, after the onset of muscle formation, some of the WHPs located towards the body cavity arrange themselves in more or less regular horizontal rows beneath the growing muscle cells. Additionally, two irregular rows of cells are formed that dorsally extend the dorsal most wing heart muscle cell (Fig. $6 \mathrm{~A})$. During the subsequent developmental steps, the cells of the epithelium begin to change their cell shape from round to discoidal. This shape change also affects their nuclei, which likewise become flat and slightly larger than the nuclei of the remaining WHPs. However, a tight contact between these cells is only established after muscle development is almost completed (after 35 h APF). Until then, gaps are still visible between the cells (Fig. 6A) and the cells are able to alter their position with respect to one another. Moreover, cell divisions may still occur or a cell from the epithelium is removed and the gap is closed by relocation of the surrounding cells (Fig. 6D).

So far, the only known reporter that specifically marks the epithelium at later stages is prc-Gal4. Initially, prc-Gal4driven $U A S-e G F P$ is detected in all WHPs from the embryo until pupal stages. At about 30-40 h APF expression becomes more prominent in the cells of the epithelium and later persists only in the epithelium (Fig. 10) 

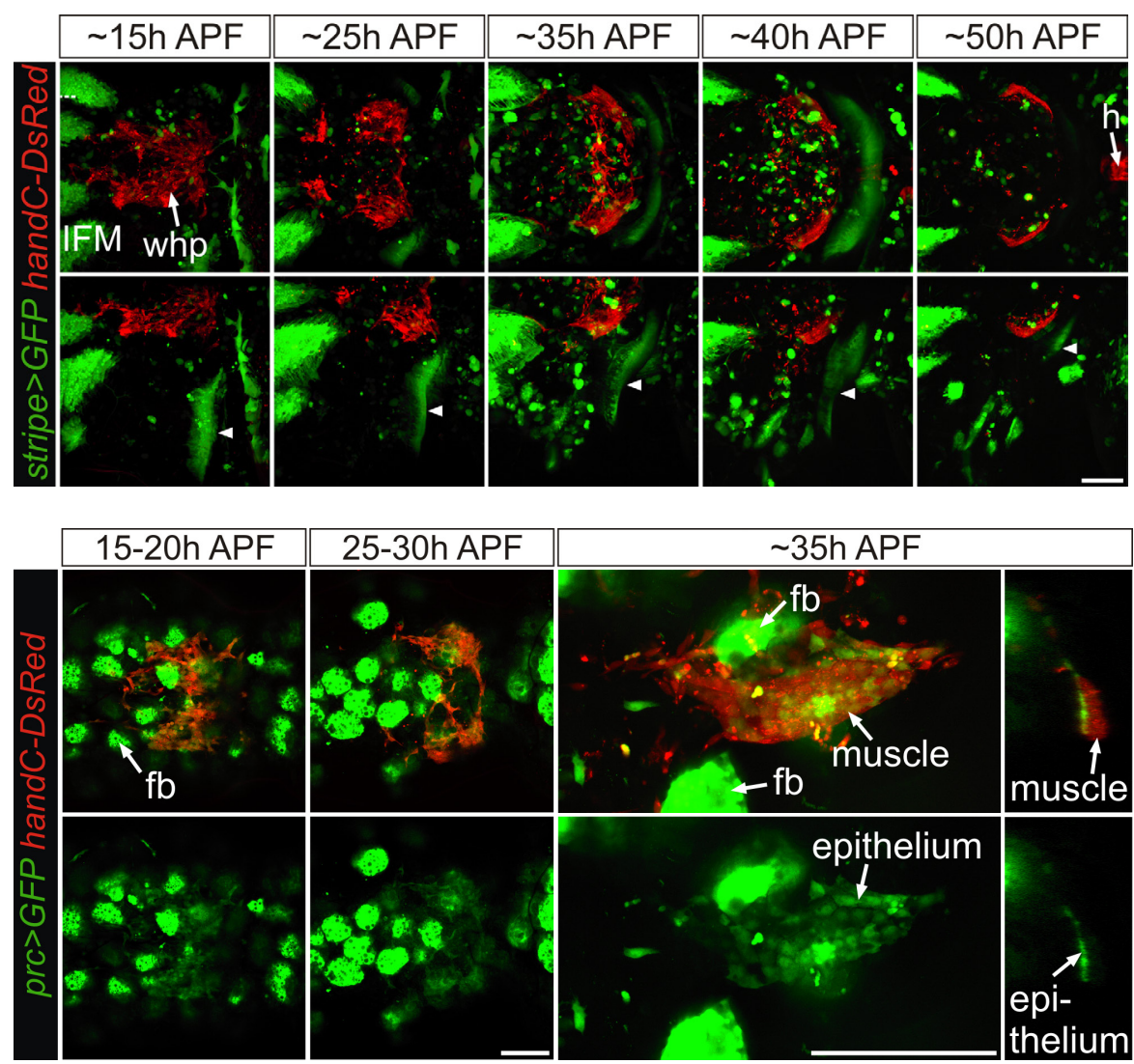

A

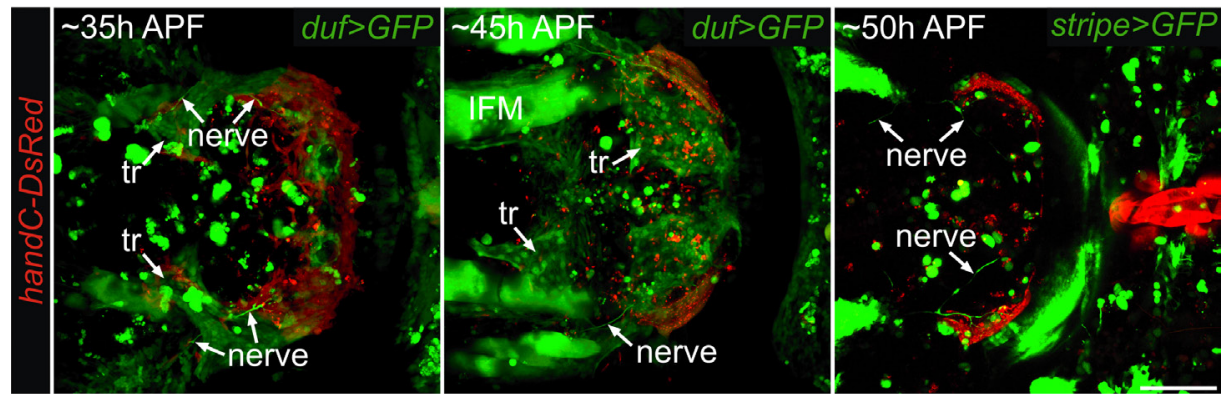

B
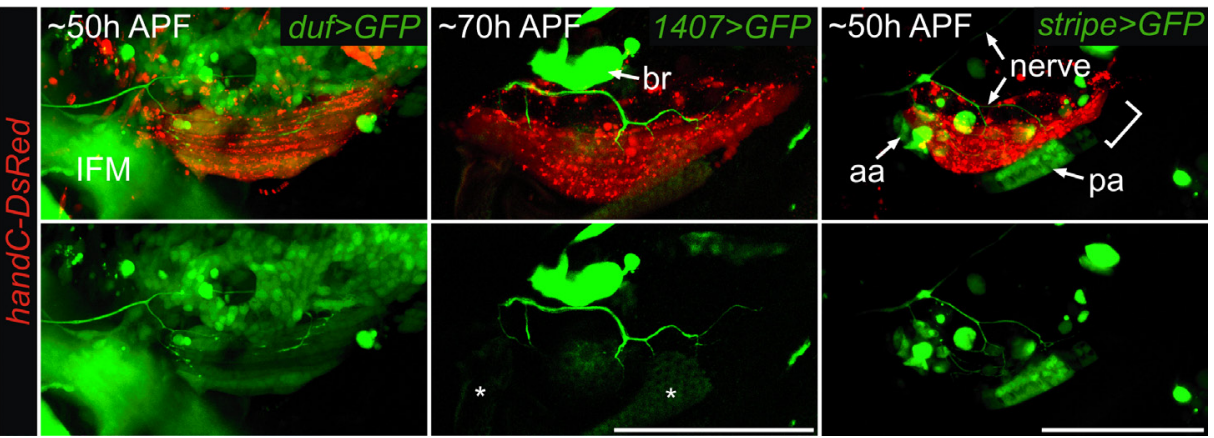

Fig. 9. Expression of stripe-Gal4 during wing heart development. The epidermal attachment sites of the indirect flight muscles (IFM) strongly express stripe-Gal4 during their entire formation (the posterior most IFMattachment site is indicated by an arrowhead). In contrast, no expression is detectable in the anterior or posterior attachment sites of the wing heart muscle cells until their development is completed at about $50 \mathrm{~h}$ APF. However, some tendon cells express either no GFP or only at very low levels. Note that highly mobile macrophages are also labeled by stripe-Gal4>UAS-GFP (green cells in the middle of the pictures). whp-wing heart progenitors, $h$ - heart. Scale bar: $100 \mu \mathrm{m}$.

Fig. 10. prc-Gal4specifically marks the wing heart epithelium at later stages. Between 30-40 h APF, the initial expression of prc-Gal4 in all wing heart progenitors (shown at 15-20 h and 25-30 h APF) becomes restricted to the wing heart epithelium. A faint expression is still detectable in the wing heart muscle cells at about 35 h APF. fb - fat body. Scale bars $100 \mathrm{um}$.
Fig. 11. Innervation of wing hearts. (A) Dorsal views of different stages of wing heart development. At about 35 h APF, duf-Gal4 marks a structure resembling a nerve that projects from the lateral side between the indirect flight muscles (IFM) to the wing hearts. Due to duf-Gal4 expression in parts of the overlying epidermis and underlying developing tracheal sacs (tr), the structure is hardly visible. stripe-Gal4 also marks this structure but the expression is very weak at early stages. (B) Lateral views of mature wing hearts with the structure marked by three different Gal4 lines (duf-Gal4, 1407-Gal4, and stripe-Gal4). $1407-G a l 4$ is expressed in the peripheral nervous system indicating that the structure is indeed a nerve. The asterisks indicate autofluorescence of the cuticle. The bracket in the top right picture marks a wing heart muscle attachment site with very low or no epidermalstripe>GFP expression. br-bristle cell, aa - anterior attachment site, pa - posterior attachment site. Scale bars $100 \mu \mathrm{m}$.

\section{Wing hearts are innervated by a peripheral nerve}

When using duf-Gal4 or stripe-Gal4 as driver line for GFP or DsRed in mature wing hearts, additional expression is detected in a nerve (Fig. 11), which projects from a lateral region located between the posterior ends of the IFMs into the scutellum and sends two or three branches into the wing heart, while the main branch continues towards the posterior end of the scutellum. The branches entering the wing heart branch further and contact the muscle cells (Fig. 11B). In duf>GFPanimals, the structure is visible as early as about $35 \mathrm{~h}$ APF and already has made contact with 
the forming wing heart (Fig. 11A). At earlier stages, the structure is hardly visible due to interfering reporter expression in surrounding tissues. In order to verify that this structure is indeed a nerve, we used Gal4 driver specific for the nervous system to express UASeGFP. While elav-Gal4 showed no expression (data not shown), the enhancer trap line 1407-Gal4, which is active in most neurons of the central nervous system and all neurons of the peripheral nervous system (Luo et al., 1994), shows a signal in this structure. Expression is first detected at about 50-60 h APF after wing heart formation is completed. For this reason, this driver line is also not suitable to study the innervation process during wing heart development. However, it supports the idea that, like all somatic muscles, wing heart muscles are innervated by motoneurons.

\section{Discussion}

Wing heart development encompasses the specification of embryonic progenitors, a dormant period, proliferation, spatial reorganization, and finally differentiation into muscular and epithelial tissues. In this study, we have focused on the pupal stages, following head eversion, during which final differentiation and maturation takes place.

\section{The role of instructive signals from surrounding tissues in wing heart development}

WHPs predominantly interact with two different types of tissue during their development: the tracheal system and the adult epidermis. The interaction with the tracheal system is established after WHP specification during embryogenesis and is especially important during the larval and prepupal stages. Furthermore, all WHPs are interconnected by cellular extensions. Since WHPs give rise to adult tissues, we suggest that both connections serve to keep the WHPs together and in close proximity to their future target site. Similarly, adult muscle precursors (AMPs) are interconnected by cellular extensions (Figeac et al., 2010) and are either associated as adepithelial cells with the imaginal discs or with peripheral nerves (Bate et al., 1991). We further suggest that WHPs are immobile during their connection with the trachea. This is based on the observation that WHPs display multiple cellular extensions by the end of embryogenesis, probably used to establish contact with one another and with the tracheal branches. These extensions are greatly reduced in number at late larval stages and even after onset of proliferation (Figs. 4A, E6). This relative lack of extensions is reflected in the smooth surfaces of the eight clusters of WHPs. This is further supported by the observation that WHPs are passively relocated together with the tracheal branches during the prepupal stage. However, with the onset of the pupal stage following head eversion, the WHPs detach from the tracheae and display multiple cellular extensions again. Asimilar quiescent phase occurring in AMPs has been interpreted as stem cell-like feature related to vertebrate satellite cells (Figeac et al., 2007). This raises the question whether the tracheae serve as a kind of niche keeping the cells dormant and activating them by their break-down at the onset of metamorphosis or if they are mere vehicles, preventing the cells from being separated from their target site by the hemolymph flow. However, the fact that not all WHPs are directly attached to tracheae argues in favor of the latter.

The second tissue that plays a crucial role during wing heart development is the adult epidermis. It serves as attachment for the WHPs after they have become free from the tracheae and also constitutes the target site where the mature wing hearts are located and where permanent connections are established in form of myotendinous junctions or the attachment of the epithelium. In contrast to the larval or prepupal stage, WHPs appear active and motile during the pupal stage. Nevertheless, since the adult epidermis undergoes massive shape changes to give rise to complex three-dimensional structures such as the scutellum, WHPs may again be passively transported. However, several observations indicate that WHPs are motile and actively migrate towards their target site. (1)WHPs exhibit multiple cellular extensions and change position in relation to one other. (2) Using the large bristle cells as landmarks within the epidermis, WHPs also move in relation to these cells (Fig. 3A). (3) Only a few WHPs are located beneath the en-Gal4 expression domain after head eversion, while the mature organs completely reside beneath this domain (Fig. 3B). The pnr-Gal4 and en-Gal4 expression domains overlap along the posterior and lateral margin of the scutellum at mid-pupal stages (Fig. 3C). However, shortly after head eversion, the lateral regions of the overlap lie more ventrally and thus more distantly from the WHPs. During the subsequent development, the lateral overlaps are relocated dorsally and form the lateral corners of the scutellum where the mature wing hearts are located. We propose that in addition to the lateral migration of WHPs, their future target site is relocated towards them during the reshaping of the epidermis. Furthermore, the lateral overlaps of the pnr-Gal4 and en-Gal4 domains may act as signaling centers that provide guiding cues to attract the WHPs.

Other regions of interaction within the epidermis are the muscle attachment sites, which are marked by stripe-Gal4. In case of the de novo forming DVMs, stripe-lacZ positive cells are already visible in the imaginal discs indicating that these cells are determined prior to muscle formation. Using stripe-Gal4 we observe a very bright staining in the epidermal attachment sites of the IFM (Fig. 9). However, no stripe-Gal4 expression was visible in the areas of the wing heart attachment sites before muscle formation was completed. Moreover, the GFP signal differed in strength between individual tendons cells and some were even stripe-Gal4 negative (Fig. 11B). This suggests that, in contrast to the IFM, the reporter is activated very late in wing heart tendon cells and also at varying levels. It is unclear whether the observed reporter expression reflects the endogenous expression of stripe in wing heart tendon cells.

\section{Scutellar arm formation: A new mode of tubulogenesis?}

Scutellar arms are tubular structures formed by the lateral epidermis to connect the scutellum with the posterior wing veins in all winged insects. In adult Drosophila, this tube is formed by a cuticular ridge and separated from the posterior notal wing process by a septum. In this study, we found that the scutellar arm develops by an outward folding of the epidermis, which creates the ridge. In the beginning, the lumen is only discernible as a thin line along the center of the folding. Later on, brightly fluorescent act5C>GFP cells are found in the center corresponding to the final diameter of the lumen. At the end of tubulogenesis, these cells migrate out of the tube and the scutellar arm is functional. The initial thin lumen seems to be the consequence of the u-shaped folding which brings neighboring epidermal areas in an opposing position leaving only a very narrow space in between. The closure of the tube towards the body cavity, and thus the formation of the septum, is probably 
achieved by a tight attachment of opposing epidermal cells at the base of the ridge. Since this folding already has a lumen, the role of the brightly fluorescent cells remains unclear. Nevertheless, their presence in the center of the forming scutellar arm suggests an involvement in lumen formation. A major difference between the initial folding and the mature scutellar arm is the thickness of the body wall marked by the reporter, which is several times thinner in the mature tube (Fig. 2C). In adult flies, the epidermis of the scutellar arm is likewise a thin layer of cells (Lehmacher et al., 2009). Since the used act5C-Gal4 line is ubiquitously expressed it was not possible to discern whether the initial thick body wall consists only of epidermis. However, one possible explanation for the occurrence of the brightly fluorescent cells might be that the epidermis undergoes a shape change from columnar to discoidal to increase the lumen of the scutellar arm. As a consequence, some epidermal cells would have to leave the epithelium due to limited space. These cells could either actively leave the tube by migration or are passively removed by macrophages. In both cases motile cells would be present in the center of the forming scutellar arm as observed in this study. Comparing the observed mode of tubulogenesis with other described modes (reviewed in Hogan and Kolodziej, 2002; Baer et al., 2009; Andrew and Ewald, 2010), it seems that it contains completely new elements. Since the epidermis is an already polarized epithelium, the formation of the ridge could be compared to a process called wrapping found in vertebrate neural tube formation. However, the outward folding of the scutellar arm leads to a tube with the apical side of the cells facing the exterior while in vertebrates it is an inward folding with the apical side facing the lumen. Furthermore, the tube remains within the epithelium and is not separated by the closure. On the other hand, the fluorescent cells in the center remind of tubulogenesis by cavitation (Baer et al., 2009; Andrew and Ewald, 2010). During this process, a cylinder of cells is hollowed by apoptosis of the cells in the center. However, this mode occurs only in non-polarized cells and likewise leads to a tube were the apical side faces the lumen.

\section{The wing heart epithelium represents a novel type of meso- dermal epithelium}

The first epithelium to arise in Drosophila is the blastoderm established during cellularization. Most of the epithelia formed later are directly derived from this first epithelium and are referred to as primary epithelia (e.g., the larval and adult epidermis, the foregut, the hindgut, the trachea, and the salivary glands). In contrast, epithelia that are formed from non-polarized mesenchymal cells by mesenchymal-epithelial transition are called secondary epithelia (e.g., the midgut, the blood-nerve barrier, the follicle cell epithelium, and the heart). Mesodermally derived secondary epithelia known so far are the heart and the follicle cell epithelium (Tepass, 1997; Tepass and Tanentzapf, 2001). Although mesodermal cells also contribute as stellate cells to the renal tubes, they only integrate into a pre-existing tube epithelium (Campbell et al., 2010). Thus, the wing heart epithelium represents a novel mesodermally derived secondary epithelium that differs morphologically from the heart and the follicle cell epithelium. While the latter form tubes, the wing heart epithelium consists of extremely flat cells that are organized in a sheet (Lehmacher et al., 2009). This is probably due to reduce mass since the epithelium is moved by muscles during beating cycles.
Time lapse movies of wing heart development indicate that wing heart epithelium formation starts after the initialization of muscle development. More importantly, tight cell-cell contacts between the cells of the epithelium are not established before wing heart muscle formation is completed. Until then the cells still change their position, divide, or are removed. During the formation of the midgut epithelium, it has been shown that the visceral mesoderm is required as substratum. Only endodermal cells that are in contact with the mesoderm undergo mesenchymalepithelial transition and develop into an epithelium (Tepass and Hartenstein, 1994). Based on the observed delay between wing heart muscle and epithelium formation, a similar mechanism might be in place during wing heart epithelium formation. In the proposed scenario, the epithelial cells require the forming muscle cells as templates or inductive signal to undergo mesenchymalepithelial transition. Subsequently, the polarized cells are able to establish tight cell-cell contacts between each other resulting in a functional epithelium.

\section{Wing heart muscle formation resembles adult myogenesis but exhibits also unique features}

Wing hearts are genuine adult organs without larval predecessors. Therefore, all myotubes are formed de novo during metamorphosis from WHPs that have been set aside during embryogenesis. This mode of myogenesis resembles the one known from adult somatic muscles, which develop from adult muscle precursors (AMPs) that likewise are specified in the embryo. Although WHPs differ in their gene expression from AMPs, they share many similarities with AMPs regarding their lineage and function. Both types of progenitors arise as siblings of embryonic muscle progenitors, proliferate after a dormant phase, and give rise to a pool of cells from which adult muscles arise. Furthermore, mature wing heart muscles resemble adult somatic muscles more closely than any other type of muscle (Lehmacher et al., 2009). In Drosophila, somatic muscle formation is based on the specification of founder cells (FC) that fuse with fusion competent myoblasts (FCM) to form multinucleated muscles (reviewed in e.g. Haralalka and Abmayr, 2010; Rochlin et al., 2010; de Joussineau et al., 2012). This mechanism is found during both the embryonic wave of myogenesis, which gives rise to the larval body wall muscles as well as the pupal wave, which forms the adult body wall muscles. In the embryo, FCs are marked by the expression of the gene dumbfounded (duf) which encodes a transmembrane receptor required for myoblast fusion (Ruiz-Gomez et al., 2000). During adult myogenesis it was found that the lacZ enhancer trap in the duf gene (Nose et al., 1998) is likewise expressed in founder cells (Kozopas and Nusse, 2002; Dutta et al., 2004; Fernandes and Keshishian, 2005). However, duf-lacZ expression is initially found in all AMPs at a low level in third instar larvae and during early pupal stages. Between 16 and $20 \mathrm{~h}$ APF this expression becomes restricted to the FCs which upregulate duf-lacZ. In this study, we have shown that WHPs likewise form a pool of cells in which duf-Gal4 positive cells are detectable from $20 \mathrm{~h}$ APF on. Subsequently, the duf-Gal4 positive cells form clusters with one or two duf-Gal4 negative cells, which represent starting points of muscle formation. Nuclei that are in close contact with duf $>$ DsRed positve nuclei become likewise DsRed positive. This can be explained by two different scenarios: (1) all nuclei initialize duf-Gal4 expression independently at dif- 
ferent times, or (2) nuclei take up DsRed because they share the cytoplasm with a duf-Gal4 expressing nucleus, e.g. after fusion. Although we were not able to directly observe fusion events in our live imaging, we propose that the initial clusters of two to three cells correspond to the described first phase of myoblast fusion and the elongation into rows to the second phase (reviewed e.g. in Önel and Renkawitz- Pohl, 2009). Since we did not specifically analyze the expression prior to 15-18 h APF, we do not know if duf-Gal4 is initially expressed in all WHPs at low levels. However, duf-Gal4 expression was detectable at very low levels in some WHPs preceding founder specification, which might correspond to the decline of expression observed in adult myogenesis.

However, in contrast to embryonic and adult myogenesis where fiber number is regulated by the number of founder cells, wing heart muscle formation comprises cell removal in addition to founder cell specification. Starting points of muscle formation occur in a broad area including the dorsal region where no muscle fibers are present in mature wing hearts (Fig. 6A). Also in this dorsal region duf-Gal4 expression was found in single cells indicating that they too represent founders. However, these growing muscle fibers were removed later during development. A possible explanation for this might be that the available space at the muscle attachment sites may be limited and only muscle cells that are able to establish contact persist. This mechanism would favor the ventral muscle fibers since they are located within the stretch of the attachment sites. An alternative explanation would be that the growing myotubes depend on innervation and that all muscles that are not contacted by the identified nerve degenerate. A similar effect has been shown in denervation experiments during the formation of the dorso-ventral muscles (DVM). In these experiments, the initial specification and position of the duf-lacZ positive founder cells was unaffected, but the later duflacZ expression was lost resulting in muscle defects (Fernandes and Keshishian, 2005). Both mechanisms have the potential to function independently of founder cell specification and may be superimposed on a tightly regulated founder cell specification network. This would explain why mature organs exhibit a varying number of muscle cells despite the occurrence of founder cells (Lehmacher et al., 2009) (Fig. 7).

Another prominent feature of wing heart muscle formation is the extensive nuclear movement that occurs in nascent fibers during development. The movement starts immediately after cluster formation and becomes more directed with the elongation of the muscle cells. During later stages, nuclei move densely packed along the antero-posterior axis of the cells (Fig. 7). A similar movement of nuclei has not been described in Drosophila so far, neither during the formation of the larval set of somatic muscles in the embryo, nor during adult myogenesis in the pupa. However, it was described to typically occur in cultured young chick muscle fibers (Capers, 1960; Englander and Rubin, 1987). Nevertheless, the function remains unclear.

Taken together, the expression of duf-Gal4 in single cells before muscle formation and the observed aggregation of cells into cluster and later rows, further supports the idea that wing heart muscle formation resembles adult somatic myogenesis and that WHPs are similar to AMPs. However, wing heart muscle formation also shows unique features like the removal of growing myotubes and the nuclear movement during development. It will be interesting to determine during future work whether the fiber number is strictly regulated in wing hearts and independent of cell removal or if these processes are interconnected representing a novel mechanism of fiber number regulation.

\section{Materials and Methods}

\section{Drosophila stocks}

The following stocks were used: handC-GFP(nuclear localization) and handC-DsRed (cytoplasmic localization) are expressed in all wing heart progenitors throughout their entire development. prc-Gal4 (expressed in, e.g. the fat body and wing heart progenitors) was a gift from M. Semeriva, Mef2-Gal4 (expressed in all myoblasts) was a gift from H.T. Nguyen, stripeGal4 (expressed in epidermal tendon cells involved in muscle attachment) was a gift from G. Morata, and rP298/duf-Gal4 (expressed in muscle founder cells) was a gift from S.D. Menon. 1407-Gal4 (BL8751) (expressed in nerves), 24B/how-Gal4 (BL1767) (expressed in muscles), act5C-Gal4 (BL3954) (ubiquitous expression), btl-Gal4(BL8807) (expressed in trachea), elav-Gal4(BL8765) (expressed in nerves), en-Gal4(BL6356) (expressed in a segmental pattern in epidermal cells), pnr-Gal4 (BL3039) (expressed in dorsal epidermal cells), UAS-eGFP(BL6874), UAS-mCD8::GFP(BL5137), and UAS-nDsRed (BL8547) were obtained from the Bloomington Stock Center. Gal4 driver lines were crossed to UAS-eGFP, UAS-mCD8::GFP or UAS-nDsRed to visualize wing hearts or related structures. All crosses were carried out at room temperature.

\section{Preparation and imaging}

Larvae were immobilized by dipping into hot water $\left(60^{\circ} \mathrm{C}\right)$ for $1-2$ seconds and then transferred to a drop of water on a microscope slide. A cover slip was put on the larvae supported by two piles of cover slips to prevent squeezing. Pupae were freed from their puparium with forceps and attached to a cover slip with a drop of Voltalef $10 \mathrm{~S}$ oil (Fig. 1A). The cover slip was then placed on top of two piles of cover slips fixed onto a microscope slide for imaging (Fig. 1B). For time lapse movies, a glass bottom dish was glued with its lid upside down onto a microscope slide. Pupae were then attached, as described above, to the glass bottom from the inside. The bottom was then tightly fixed into the lid with a rubber band to prevent dehydration (Fig. 1C). Epifluorescence images were obtained with a Leica MZ16FA stereomicroscope using the software package AnalySIS (SIS software, Münster, Germany). Confocal microscopy was performed using a Zeiss 5 Pascal LSM with a Plan-Neofluar 25x/0.8 objective. Images were processed using the Zeiss AIM software. Cross and horizontal sections, enlargements in Fig. 5, inset in Fig. 8, and early stages in Fig. 10 are single slices taken from confocal stacks. All other images are maximum projections generated from confocal stacks. Movies were created from maximum projections using Fiji (http://fiji.sc).

\section{Acknowledgements}

We thank H. Nuygen, G. Morata, M. Semeriva, S.D. Menon, and the Bloomington Drosophila stock center for generous gifts of stocks, and M. Biedermann and M. Krabusch for excellent technical assistance. This work was supported by grants from the DFG to A.P. (SFB 944) and the FWF to G.P. (P19380).

\section{References}

ANDREW DJ and EWALD, AJ. (2010). Morphogenesis of epithelial tubes: Insights into tube formation, elongation, and elaboration. Dev. Biol. 341: 34-55.

BAER MM, CHANUT-DELALANDE, $\mathrm{H}$ and AFFOLTER, M. (2009). Cellular and molecular mechanisms underlying the formation of biological tubes. Curr. Top. Dev. Biol. 89: 137-162.

BATE M, RUSHTON, E and CURRIE, DA. (1991). Cells with persistent twist expression are the embryonic precursors of adult muscles in Drosophila. Development 113: 79-89.

CALLEJA M, HERRANZ, H, ESTELLA, C, CASAL, J, LAWRENCE, P, SIMPSON, P 


\section{M. Tögel et al.}

and MORATA, G. (2000). Generation of medial and lateral dorsal body domains by the pannier gene of Drosophila. Development 127: 3971-3980.

CAMPBELL K, CASANOVA, J and SKAER, H. (2010). Mesenchymal-to-epithelial transition of intercalating cells in Drosophila renal tubules depends on polarity cues from epithelial neighbours. Mech. Dev. 127: 345-357.

CAPERS, C.R. (1960). Multinucleation of skeletal muscle in vitro. J. Biophys. Biochem. Cytol. 7: 559-566.

DE JOUSSINEAU C, BATAILLÉ, L, JAGLA, T and JAGLA, K. (2012). Diversification of muscle types in Drosophila: upstream and downstream of identity genes. Curr. Top. Dev. Biol. 98: 277-301.

DUTTA D, ANANT, S, RUIZ-GOMEZ, M, BATE, M and VIJAYRAGHAVAN, K. (2004) Founder myoblasts and fibre number during adult myogenesis in Drosophila. Development 131: 3761-3772.

ENGLANDER, L. L. and RUBIN, L. L. (1987). Acetylcholine receptor clustering and nuclear movement in muscle fibers in culture. J. Cell Biol. 104: 87-95.

FERNANDES JJ and KESHISHIAN, H. (2005). Motoneurons regulate myoblast proliferation and patterning in Drosophila. Dev. Biol. 277: 493-505.

FIGEAC N, DACZEWSKA, M, MARCELLE, C and JAGLA, K. (2007). Muscle stem cells and model systems for their investigation. Dev. Dyn. 236: 3332-3342.

FIGEAC N, JAGLA, T, ARADHYA, R, DA PONTE, JP and JAGLA, K. (2010). Drosophila adult muscle precursors form a network of interconnected cells and are specified by the rhomboid-triggered EGF pathway. Development 137: 1965-1973.

FRISTROM, D. and FRISTROM, JW. (1983). The metamorphic development of the adult epidermis. In The development of Drosophila melanogaster, vol. 2 (ed. BATE, M. and MARTINEZARIAS, A.). Cold Spring Harbor, New York, pp.843-897.

HARALALKA, S. and ABMAYR, S.M. (2010). Myoblast fusion in Drosophila. Exp. Cell Res. 316: 3007-3013.

HOGAN BL and KOLODZIEJ, PA. (2002). Organogenesis: molecular mechanisms of tubulogenesis. Nat. Rev. Cancer 3: 513-523.

KOZOPAS KM and NUSSE, R. (2002). Direct flight muscles in Drosophila develop from cells with characteristics of founders and depend on DWnt-2 for their correct patterning. Dev. Biol. 243: 312-325.
KRENN HW and PASS, G. (1995). Morphological diversity and phylogenetic analysis of wing circulatory organs in insects, part II: Holometabola. Zoology 98: 147-164.

LEHMACHER C, TÖGEL, M, PASS, G and PAULULAT, A. (2009). The Drosophila wing hearts consist of syncytial muscle cells that resemble adult somatic muscles. Arthropod Struct. Dev. 38: 111-123.

LUO L, LIAO, YJ, JAN, LY and JAN, YN. (1994). Distinct morphogenetic functions of similar small GTPases: Drosophila Drac1 is involved in axonal outgrowth and myoblast fusion. Genes \& Dev. 8: 1787-1802.

NOSE A, ISSHIKI, T and TAKEICHI, M. (1998). Regional specification of muscle progenitors in Drosophila: the role of the msh homeobox gene. Development 125: $215-223$

ÖNEL, S.F. and RENKAWITZ-POHL, R. (2009). FuRMAS: triggering myoblast fusion in Drosophila. Dev. Dyn. 238: 1513-1525.

PASS G. (2000). Accessory pulsatile organs: evolutionary innovations in insects Annu. Rev. Entomol. 45: 495-518.

PASS G, GEREBEN-KRENN, B-A, MERL, M, PLANT, J, SZUCSICH, NU and TÖGEL, M. (2006). Phylogenetic relationships of the orders of Hexapoda: Contributions from the circulatory organs for a morphological data matrix. Arthropod Syst. Phylogeny 64: 165-203.

ROCHLIN, K., YU, S., ROY, S. and BAYLIES, M.K. (2010). Myoblast fusion: when it takes more to make one. Dev. Biol. 341: 66-83.

RUIZ-GÓMEZ M, COUTTS, N, PRICE, A, TAYLOR, MV and BATE, M. (2000). Drosophila dumbfounded: a myoblast attractant essential for fusion. Cell102: 189-198.

TEPASS U. (1997). Epithelial differentiation in Drosophila. Bioessays 19: 673-682.

TEPASS U and HARTENSTEIN, V. (1994). Epithelium formation in the Drosophila midgut depends on the interaction of endoderm and mesoderm. Development 120: $579-590$

TEPASS $U$ and TANENTZAPF, G. (2001). Epithelial cell polarity and cell junctions in Drosophila. Annu. Rev. Genet. 35: 747-784.

TÖGEL M, PASS, G and PAULULAT, A. (2008). The Drosophila wing hearts originate from pericardial cells and are essential for wing maturation. Dev. Biol. 318: 29-37.

USUI K and SIMPSON, P. (2000). Cellular basis of the dynamic behavior of the imaginal thoracic discs during Drosophila metamorphosis. Dev. Biol. 225: 13-25. 


\section{Further Related Reading, published previously in the Int. J. Dev. Biol.}

The serpin Spn5 is essential for wing expansion in Drosophila Melanogaster

Yves Charron, Rime Madani, Chantal Combepine, Vincent Gajdosik, Yeukuang Hwu, Giorgio Margaritondo and Jean-Dominique Vassalli

Int. J. Dev. Biol. (2008) 52: 933-942

Muscle patterning and specification in Drosophila Mar Ruiz-Gomez

Int. J. Dev. Biol. (1998) 42: 283-290

dTcf antagonises Wingless signalling during the development and patterning of the wing in Drosophila

N Lawrence, P Dearden, D Hartley, J Roose, H Clevers and A M Arias

Int. J. Dev. Biol. (2000) 44: 749-756

Rab11 is required for cell adhesion, maintenance of cell shape and actin-cytoskeleton organization during Drosophila wing development

Tanmay Bhuin and Jagat K. Roy

Int. J. Dev. Biol. (2011) 55: 269-279

The ash2 gene is involved in Drosophila wing development

Montserrat Amorós, Montserrat Corominas, Peter Deák and Florenci Serras

Int. J. Dev. Biol. (2002) 46: 321-324

5 yr ISI Impact Factor $(2011)=2.959$

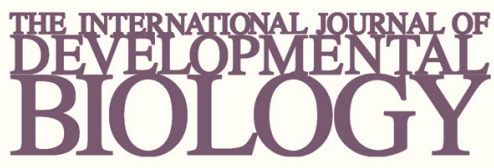

Volume 54 Nos. $6 / 7$
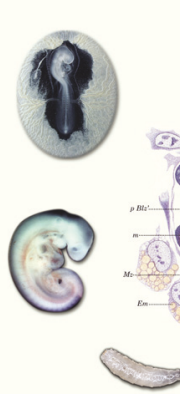

Developmental Hematopoiesis
Special Issue

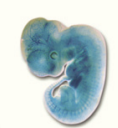

(2) (8)

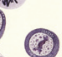

(2)
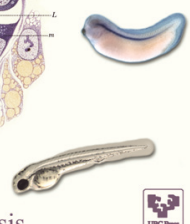

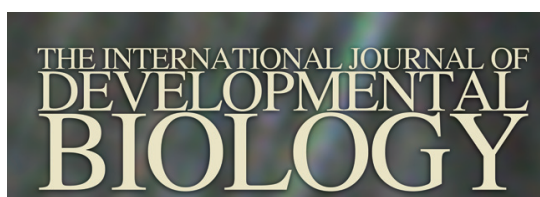

Volume 56 Nos. 1/2/3

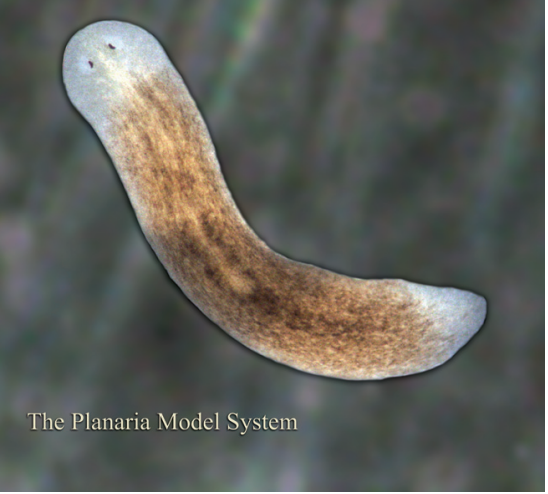

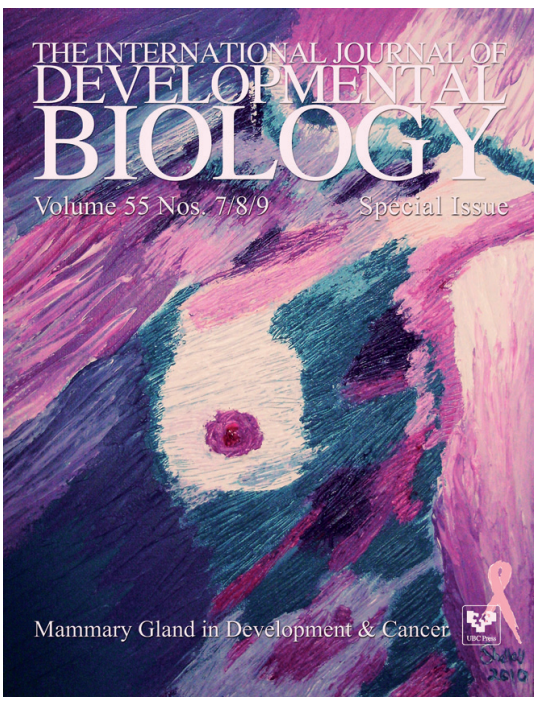

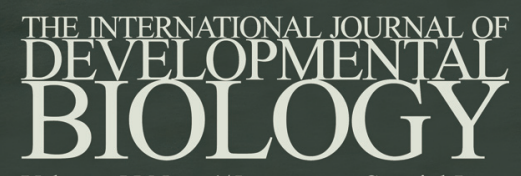

Volume 55 Nos. $4 / 5$

Special Issue

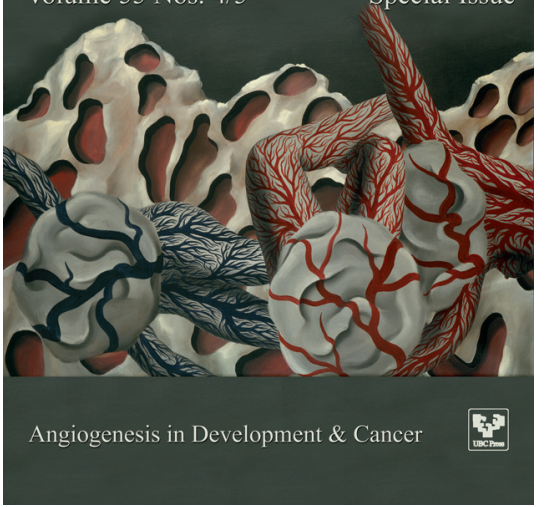

